\title{
Interoception modulates fear detection in binocular onset rivalry
}

Cassandra D. Gould van Praag ${ }^{1,2,5 *}$, Gabriel Hassan ${ }^{3}$, Ryan B. Scott ${ }^{2,3}$, Anil K. Seth ${ }^{2,4}$, Hugo D. Critchley $^{1,2}$ and Sarah N. Garfinkel ${ }^{1,2}$

${ }^{1}$ Department of Neuroscience, Brighton and Sussex Medical School, Brighton, UK.

${ }^{2}$ Sackler Centre for Consciousness Science, University of Sussex, Brighton, UK.

${ }^{3}$ School of Psychology, University of Sussex, Brighton, UK.

${ }^{4}$ Department of Informatics, University of Sussex, Brighton, UK.

${ }^{5}$ Department of Psychiatry, University of Oxford, Oxford, UK.

*Corresponding author: cassandra.gouldvanpraag@psych.ox.ac.uk

\section{Abstract}

Interoception is the body-to-brain axis of sensation concerning the state of the internal body. Interoceptive signals from the heart can modulate emotional processing. Moreover, the degree to which people accurately detect their own heart beating at rest can predict their emotional responsivity. Here we determine how interoceptive signals can modulate the perception of faces by exploiting phasic cardiac activity within a binocular onset rivalry paradigm. We demonstrate that afferent cardiac signals facilitate the early processing of fearful face images in individuals with high interoceptive (heartbeat detection) accuracy: The contraction of heart at cardiac systole enhanced the detection of fearful faces (during initial image selection in rivalrous presentations), and shortened reaction times for subsequent emotional judgements. However, cardiac systole does not lead to an overall bias in increased fear categorisation. Our findings highlight interactive contributions of interoception in shaping perceptual and behavioural aspects of emotional processing. Our results thereby add to empirical data regarding mechanisms of body-brain integration underpinning emotional experience. 


\section{Introduction}

Interoception describes the processing of signals from the internal bodily organs, including physiological changes associated with autonomic arousal (Sherrington, 1948). By extension, interoception encompasses integrated representations of bodily physiology that underpin subjective feelings and contribute to emotion in shaping cognition and behaviour (Gendron \& Barrett, 2009). Individuals vary in their sensitivity to interoceptive signals, as shown using heartbeat detection tasks to quantify the accuracy with which individuals can report sensations of their own heart beating at rest. Consistent with 'peripheral' theories of emotion (e.g. Lange \& James, 1967), sensitivity to changes in the body can modulate sensory-perceptual and subjective aspects of emotional processing. Correspondingly 'good heartbeat detectors' report enhanced subjective emotional responses compared to poor heartbeat detectors (Dunn et al., 2010; Wiens, Mezzacappa, \& Katkin, 2000).

Autonomic arousal in the cardiovascular system leads to an increase in heart rate and blood pressure. These changes are signalled to the brain via the firing of arterial stretch receptors (baroreceptors) when blood is ejected from the heart at ventricular systole into the aortic arch and carotids. Baroreceptor firing is the major interoceptive channel through which cardiovascular arousal influences brain processes, transmitting information about the strength and timing of individual heartbeats to the brainstem via cranial nerves. These arousal signals influence perceptions, thoughts, feelings and behaviours (Critchley, Eccles, \& Garfinkel, 2013). Phasic baroreceptor activation, signalling momentary cardiovascular arousal, suppresses the processing of brief nociceptive stimuli and mnemonic encoding of word stimuli (Edwards, McIntyre, Carroll, Ring, \& Martin, 2002; Garfinkel et al., 2013), but can enhance the processing of threat and fear signals (Garfinkel et al., 2014). There is still incomplete characterization of how natural baroreceptor activation modulates percept formation and conscious access of emotionally salient stimuli, though it is likely that this marker of arousal interacts with individual differences in other psychological 
facets of interoception (Garfinkel, Seth, Barrett, Suzuki, \& Critchley, 2015). Indeed, Pfeifer et al., demonstrated that individuals who score highly on tests of interoceptive accuracy show enhanced learning of fearful faces during phasic baroreceptor activation, but no enhancement of learning for neutral or happy faces (Pfeifer et al., 2017).

Binocular rivalry and dichoptic image presentations are a widely-employed approach (almost the "gold standard") in the investigation of factors that compete to modulate conscious perception (Giles, Lau, \& Odegaard, 2016). Binocular rivalry describes the perceptual phenomenon evoked when the two eyes are simultaneously presented with a different image: Due to competition over multiple levels of the visual hierarchy (e.g. Blake \& Logothetis, 2002; Tong, Meng, \& Blake, 2006), only one of the presented images tends to be consciously perceived. Binocular onset rivalry paradigms, also known as dichoptic masking paradigms (Alpers \& Pauli, 2006), involve the brief dichoptic presentation of paired stimuli; the experimental variable of interest is the image that is first perceived. This method contrasts with extended binocular rivalry presentations, since there is no transition in perception between the rivalrous images. Binocular onset rivalry may be considered an appropriate approximation of perceptual rivalry encountered in normal vision (Stanley, Forte, Cavanagh, \& Carter, 2011), and this approach is particularly well-suited to assess the influence of factors that bias the rapid formation of visual percepts (Carter \& Cavanagh, 2007).

Perceptual choice between rivalrous faces at stimulus onset is influenced by low level factors including the relative luminance contrast of images (Chong \& Blake, 2006; Song et al., 2009), their display locations (Carter \& Cavanagh, 2007) and by the interval between successive presentations (the 'removal period') (Carter \& Cavanagh, 2007; Leopold, Wilke, Maier, \& Logothetis, 2002). The initial choice selection is also influenced by the emotionality of images (e.g. Alpers \& Gerdes, 2007), attention (Chong \& Blake, 2006), perceptual memory (for a review see Pearson \& Brascamp, 2008), and by the anxiety state of the observer (K. L. H. H. Gray, Adams, \& Garner, 2009). However, there is an absence of research into how physiological arousal or individual differences in interoceptive 
abilities might affect initial image selection, and whether the enhanced processing of threat signals during baroreceptor activation is also manifest as a bias in the rapid formation of visual percepts. . Here we tested the influence of cardiac afferent signals and interoceptive ability on competitive perceptual processing in the rapid formation of conscious percepts of fearful and neutral faces. We thereby determine cardiac-mediated alterations to the intrinsic attentional salience of fearful over neutral faces (expressed as perceptual dominance of the fearful face in dichotic rivalry tasks) (e.g. Alpers \& Gerdes, 2007; K. L. H. Gray et al., 2009). To achieve this we time-locked the brief presentation of dichoptic images to specific phases of the cardiac cycle. In Experiment 1, we quantified the amount of visual degradation necessary to reverse the inherent onset selection bias of fearful over neutral faces at cardiac systole (modelling cardiovascular arousal) and separately at cardiac diastole (modelling a non-aroused state), and used this metric to measure the influence of afferent cardiac signals. We employed a staircase modulation of the visual contrast of the fearful face against a fixed contrast neutral face, ensuring that neutral faces were perceived in the majority (targeted $77.85 \%$ ) of trials. By this method, we calculated the individual contrast threshold ('fear sensitivity') required for minimal conscious access of the fearful face at systole and diastole, respectively when cardiac afferents are activated and quiescent. Importantly, we determined whether there were differences in fear sensitivity for differing individual levels of interoceptive ability. Following previous findings showing that fear sensitivity is augmented at cardiac systole (Garfinkel et al., 2014; Garfinkel \& Critchley, 2016), we hypothesised that stimulus presentation at cardiac systole would enhance the processing of threat stimuli, as demonstrated by enhanced fear sensitivity at systole relative to diastole. Moreover, as interoception is related to emotionality (Dunn et al., 2010; Wiens et al., 2000) we hypothesised that interoceptive abilities would modulate the impact of cardiac timing on percept formation, with individuals with the greatest interoceptive abilities manifesting an amplification of the differential effects of cardiac phase. In Experiment 2, which followed immediately from Experiment 1, we determined whether enhanced sensitivity in the detection of threat signals, as a function of cardiac phase and interceptive ability, would bias the 
categorisation of ambiguous emotional faces presented at the threshold of fear detection. Here we hypothesised that cardiac systole and high interoceptive abilities would also be associated with an increased tendency to categorize ambiguous faces as fearful, demonstrating and assessing the behavioural impact of enhanced sensitivity to the detection of threat stimuli hypothesised for Experiment 1.

\section{Experiment 1: Fear Sensitivity}

\section{Method}

\section{$\underline{\text { Participants }}$}

Before beginning data collection, we decided on a sample size which matched those previously used in the detection of cardiac effects (Garfinkel et al., 2013; Garfinkel et al., 2014), with increased numbers to enable the detection of a potentially smaller effect via this untested method. Thirty participants were included in the study ( 23 female, mean age $=29.2$ years, $s d=10.5$ years). All participants had normal or corrected to normal vision and gave written informed consent. Ethical approval for the conduct of this investigation was provided by the Brighton and Sussex Medical School. All procedures were carried out in accordance with the provisions of the World Medical Association Declaration of Helsinki.

\section{Procedure}

Fear sensitivity

Face stimuli were adapted from the Karolinska Directed Emotional Faces (KDEF) set (Lundqvist, Flykt, \& Ohman, 1998). Twenty-six fear and neutral image pairs were selected from the set, with the same KDFE-model showing the fear and neutral expression in each pair (13 male and 13 female pairs) The same KDFE-model pairing was maintained throughout Experiments 1 and 2. All images where converted to greyscale and luminance matched across pairs. A black oval mask was applied to cover 
hair, and the eyes were aligned to a fixed and equal position across all images. Images were surrounded by black and white square contours to aid fusion of the binocular images and subtended 6.87 degrees of visual angle. For each trial, one pair from the 26 was randomly selected for presentation.

Participants viewed stimuli through a mirror stereoscope, supported by a chin rest. The stereoscope and image position were calibrated to achieve stable image fusion for each participant prior to beginning the experiment. Participants were informed that a fearful or neutral face would be briefly presented, and that they should categorise the perceived emotion as "Fear" or "Neutral" by using their dominant hand to press the left or right arrow key respectively. Participants were instructed to report their percept as quickly and accurately as possible. No time limits were imposed, progression between trials was self-paced and reaction times not recorded. All participants completed between one and three practice trials.

In each trial, participants were presented with a fearful face in their non-dominant eye, and the matched neutral face in their dominant eye. Eye dominance was determined using the Miles test (Miles, 1930). Participants were instructed to overlap their hands with thumbs facing outwards, creating a small gap subtended by the thumb and forefinger of both hands. The participant then views a distant object through the gap with their arms outstretched and both eyes open, and naturally positions the viewing gap over the eye with functional dominance. The non-dominant eye was selected for presentation of the fearful face to minimise the potential for ceiling effects associated with the initial selection preference of emotional images (Alpers \& Gerdes, 2007). Images were presented for $100 \mathrm{~ms}$, time locked to cardiac systole or diastole as determined via concurrent electrocardiographic (ECG) recording. ECG data were acquired using a standard three lead configuration and recorded via a Cambridge Electronic Design (CED) 1902 amplifier and a CED Power 1401 mkll waveform convertor for digital output. Images were presented on the R wave for diastole 
trials and $\mathrm{R}+300 \mathrm{~ms}$ for systole trials, to coincide with the T wave when baroreceptors are processed centrally (Edwards, Ring, McIntyre, Winer, \& Martin, 2009).

Sensitivity to fearful stimuli was calculated via staircase modulation of the contrast of the fearful image (Figure 1). Fear faces were initially degraded to $10 \%$ contrast, and then modulated according to a fixed one-down-one-up step rule using a ratio of $\delta$-/ $\delta+$ of 0.2845 over 20 reversals. In simulations this ratio has been shown to converge on a detection rate of $22.15 \%$ in two alternateforced-choice paradigms when the spread of the psychometric function is known (García-Pérez, 1998), thus the staircase should produce a fear categorisation of approximately $22.15 \%$ in the present investigation. This detection rate was determined to be the most suitable level from the range identified as stable by García-Pérez (1998) (14.16\%, 16.85\%, 19.65\%, 22.15\%), to minimise the likelihood of ceiling effects in Experiment 2 in which we expected the detection rate to be modulated up or down by systole or diastole respectively.

The contrast of the neutral face was maintained at $100 \%$ throughout. Fear sensitivity was calculated as $100 \%$ minus the mean contrast of the fearful face over the final 16 reversals. Two staircases were run independently and simultaneously, each locked consistently to systole or diastole, with trials of each staircase randomly interleaved. 


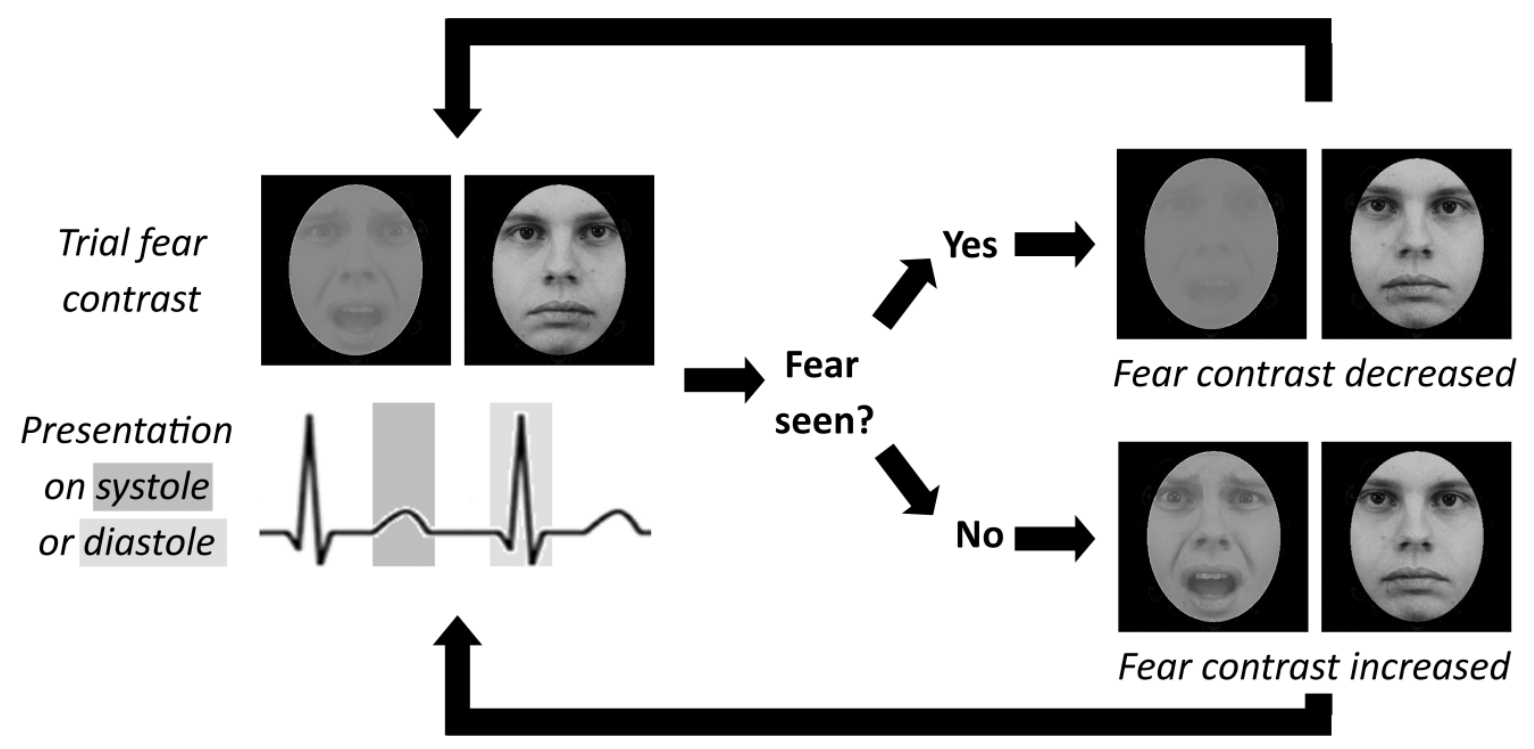

Figure 1: Staircase procedure used to determine fear sensitivity. Two separate staircases were each locked consistently to systole or diastole, with trials randomly interleaved between the two. In all trials, a full contrast neutral face was presented to the dominant eye, while a fearful face was presented to the non-dominant eye at a contrast determined by the preceding trials.

We minimised the influence of perceptual memory effects in Experiment 1 by using a large stimulus set with minimal repetitions of the same face (Stanley et al., 2011). We also promoted the likelihood that participants would readily be able to switch between the fearful and neutral percepts (i.e. override perceptual memory of either the fearful or neutral face) by using short presentation durations (100 ms) with long inter-trial-intervals (ITI) (mean ITI = $6.05 \mathrm{~s}, \mathrm{sd}=0.91 \mathrm{~s}$ ), which have previously been show to generate switching in onset rivalry (Carter \& Cavanagh, 2007; Stanley et al., 2011).

\section{Interoception and anxiety}

Interoceptive accuracy (Garfinkel et al., 2015) was determined using a heartbeat tracking task (Schandry, 1981), modified to use a finger pulse oximeter ('soft' mount PureLight sensor; Nonin Medical Inc., MN, USA) to record heartbeat data rather than ECG. Participants were instructed to focus on their heartbeat sensations without active tactile arterial palpation, and count the number of heartbeats they felt over fixed durations of $25 \mathrm{~s}, 30 \mathrm{~s}, 35 \mathrm{~s}, 40 \mathrm{~s}, 45 \mathrm{~s}$ and $50 \mathrm{~s}$ (presented in a 
randomised order). Heartbeat data were recorded concurrently, and interoceptive accuracy determined for each trial through comparison between the number of beats reported and the number beats objectively recorded as per equation 1. Accuracy scores were averaged over the 6 trials, yielding an accuracy value for each participant (Hart, McGowan, Minati, \& Critchley, 2013).

Interoceptive accuracy per trial $=1-\frac{\mid \text { nbeats }_{\text {recorded }}-\text { nbeats } \text { reported } \mid}{(\text { nbeats recorded }+ \text { nbeats } \text { reported }) / 2}$

Participants were ranked by interoceptive accuracy and median split into "low interoceptive accuracy" ( $n=15 ;$ mean accuracy $=32.7 \% ; s d=2.3 \%)$ and "high interoceptive accuracy" $(n=15$; mean accuracy $=83.4 \% ; \mathrm{sd}=1.05 \%$.

State anxiety was assessed immediately before the experimental session, using the "state" subscale of the Spielberger State-Trait Anxiety Inventory (Spielberger, Gorsuch, Lushene, Vagg, \& Jacobs, 1983).

\section{Analysis}

\section{Stimulus timing}

The distribution of stimulus timings (mean and standard deviation) were calculated for each participant, to ensure that trial events were appropriately time-locked to cardiac systole (ECG Rwave) or cardiac diastole (ECG T-wave: R-wave $+300 \mathrm{~ms}$ ) (see Figure $2 \mathrm{~A}$ for mean stimulus times achieved). One participant was excluded as the standard deviation of stimulus timings for systole and diastole exceeded the range of the group mean $\pm 2 \mathrm{sd}$ (mean systole standard deviation $=61.52$ $\mathrm{ms}(\mathrm{sd}=45.35 \mathrm{~ms}) ;$ mean diastole standard deviation $=58.22 \mathrm{~ms}(\mathrm{sd}=33.92 \mathrm{~ms})$. 


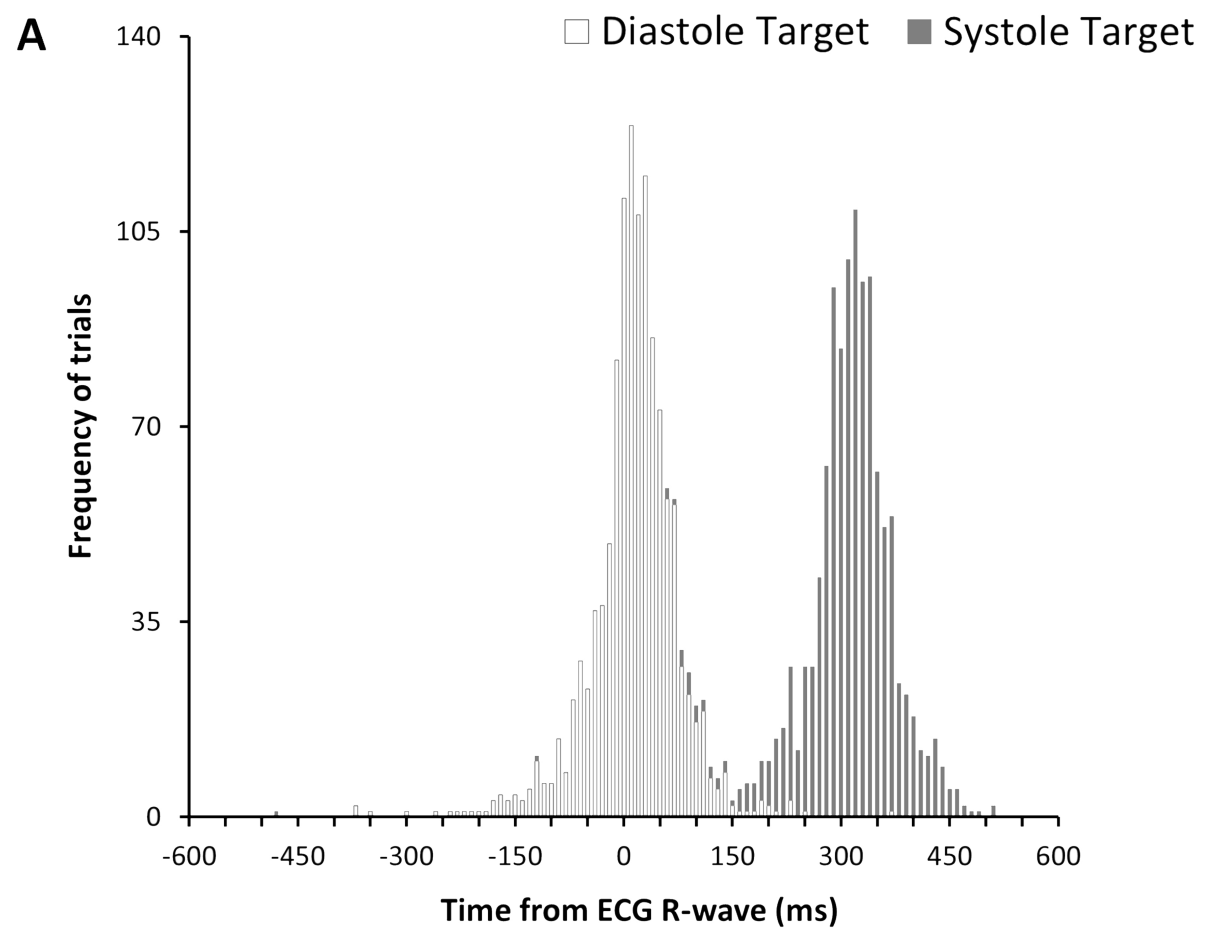

B $\quad 2607 \quad \square$ Diastole Target $\square$ Systole Target

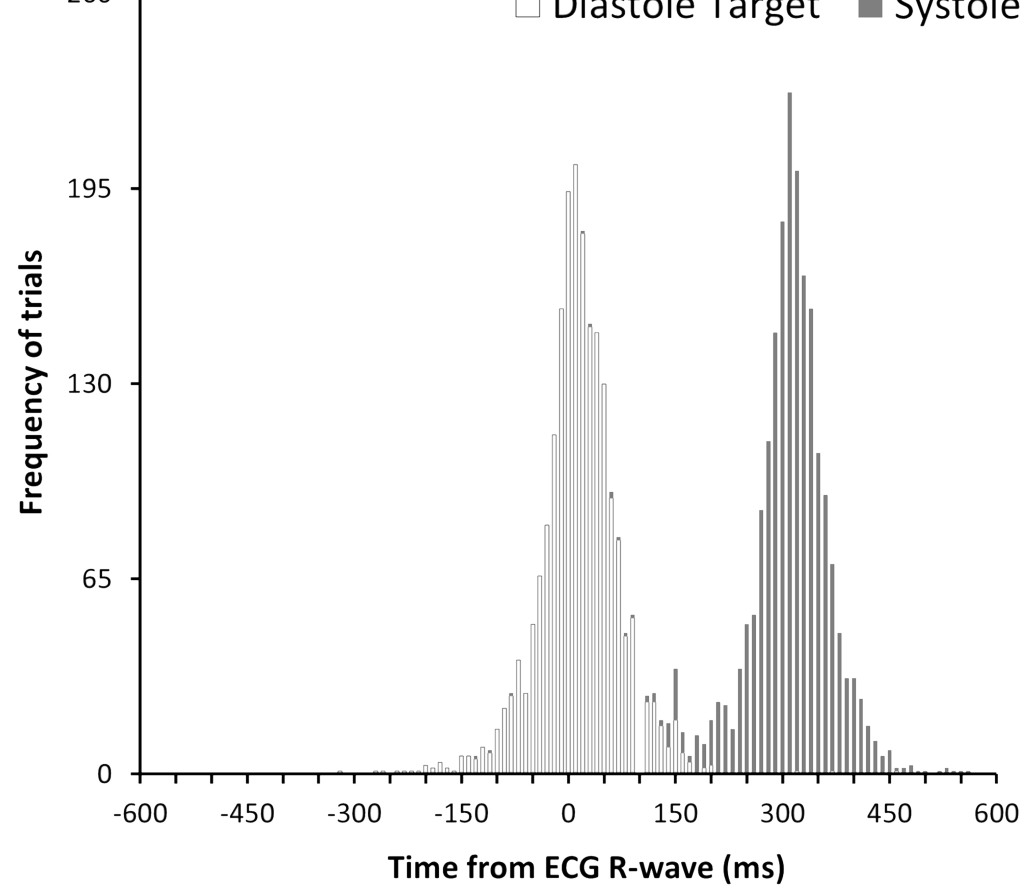

Figure 2. Frequency of systole and diastole trial times for all included participants with respect to the ECG R-wave. (A) Experiment $1(n=29)$ : diastole mean time $=R+14.21 \mathrm{~ms}$, sd $=56.92 \mathrm{~ms}$; systole mean time $=R+313.83 .42 \mathrm{~ms}, \mathrm{sd}=58.30 \mathrm{~ms}$. (B) Experiment $2(n=28)$ : diastole mean time $=R+$ $11.03 \mathrm{~ms}, \mathrm{sd}=55.62 \mathrm{~ms}$, systole mean time $=\mathrm{R}+310.82 \mathrm{~ms}, \mathrm{sd}=57.55 \mathrm{~ms}$. 


\section{Statistical analysis}

All data were normally distributed according the Shapiro-Wilk test statistic $(p>.05)$. To assess differences in the image contrast required to reverse the onset bias of fearful over neutral faces at systole and diastole (Experiment 1), fear sensitivities for systole and diastole were entered into a two-way mixed ANCOVA with the between subjects factor "interoceptive accuracy" (low, high) and covariate state anxiety. Cardiac effects are expressed as the difference between systole and diastole (systole - diastole), with alpha set at .05. All statistics are reported with two-tailed significance. Effect sizes are presented as partial eta-squared statistics, denoting the variance explained by the variable after removing the variance associated with other predictors $\left(\eta_{p}^{2}=.02\right.$ small effect; $\eta_{p}^{2}=.13$ medium effect; $\eta_{p}^{2}=.26$ large effect).

\section{Results and discussion}

There was no overall main effect of cardiac timing on fear sensitivity $\left(F(1,25)=0.83, p=.371, \eta_{p}^{2}=\right.$ .03), indicating that, across all participants, systole and diastole did not impact the relative perceptual dominance of fear over neutral signals. However, we observed the predicted significant interaction between cardiac phase and interoceptive accuracy $\left(F(1,25)=8.20, p=.008, \eta_{p}^{2}=.25\right)$, evident as a significant difference in fear sensitivity at systole and diastole between high and low interoceptive accuracy groups (mean difference $=-7.95 \%, 95 \% \mathrm{Cl}[-13.73,-2.17] ;(t(26)=2.83, p=$ $.009, \eta_{p}^{2}=.24$, Figure 3A). Individuals with high interoceptive accuracy showed increased fear sensitivity at systole compared to diastole (mean systole fear sensitivity $=80.14 \%$ ( $s d=14.00 \%$ ), mean diastole sensitivity $=75.27 \%(s d=15.94 \%) ;$ mean systole - diastole difference $=4.977 \%, 95 \%$ $\mathrm{Cl}[0.83,9.11])$. In contrast, individuals with low interoceptive accuracy showed a non-significant reduction in fear sensitivity at systole compared to diastole (mean systole fear sensitivity $=72.36 \%$ $(s d=16.06 \%)$, mean diastole fear sensitivity $=75.44 \%(s d=18.04 \%) ;$ mean systole - diastole difference $=-3.19 \%, 95 \% \mathrm{Cl}[-7.32,0.95])$. There were no significant interactions between state anxiety and overall fear sensitivity or cardiac phase (all $p>.05$ ). 
These data suggest that participants with high interoceptive accuracy were more sensitive to the detection of fearful faces when they were presented at systole relative to diastole, while participants with low interoceptive accuracy were less sensitive at systole relative to diastole. In Experiment 2 we sought to determine whether this differential sensitivity was generalised to an overall bias in the tendency to categorise emotionally ambiguous faces as fearful at systole relative to diastole, and whether this bias was also influenced by an individual's interoceptive ability.

\section{Experiment 2: Emotion categorization}

\section{Method}

\section{Participants}

Experiment 2 followed immediately from Experiment 1, and all participants of Experiment 1 also took part in Experiment 2.

\section{Procedure}

\section{Emotion categorisation}

Participants viewed matched pairs of fearful and neutral faces through dichoptic viewing apparatus, with images presented at systole and diastole as in Experiment 1. Participants were again informed that they were required to categorise the emotion perceived in the presented images ("Fear" or "Neutral") as quickly and accurately as possible. However, in Experiment 2 the contrast of the fearful face was fixed to the mean of the contrasts calculated for systole and diastole by in Experiment 1 for that participant. All other display parameters were the same as Experiment 1. Participants completed 156 trails with a fixed fear contrast, with $50 \%$ of trials locked to systole and $50 \%$ of trails locked to diastole, in a randomised order. Cardiac effects are expressed as the difference between systole and diastole (systole - diastole) in the frequency of trials classified as fear (percentage fear detection), and the time between removal of the stimulus and the key press response (reaction 
time). Effect sizes are presented as partial eta squared statistics, denoting the variance explained by the variable after removing the variance associated with other predictors $\left(\eta_{p}^{2}=.02\right.$ small effect; $\eta_{p}^{2}=$ .13 medium effect; $\eta_{p}^{2}=.26$ large effect).

\section{Interoception and anxiety}

Interoceptive and state anxiety measures determined in Experiment 1 were applied in the analysis of Experiment 2.

\section{Analysis}

\section{Stimulus timing}

The distribution of stimulus timings (mean and standard deviation) were calculated for each participant as per Experiment 1 (see Figure 2B for mean stimulus times achieved). Two participants were excluded from Experiment 2 as the standard deviation of stimulus timings for systole and diastole exceeded the range of the group mean $\pm 2 \mathrm{sd}$ (mean systole standard deviation $=55.90 \mathrm{~ms}$ $(\mathrm{sd}=21.63 \mathrm{~ms}) ;$ mean diastole standard deviation $=55.00 \mathrm{~ms}(\mathrm{sd}=25.74 \mathrm{~ms})$.

\section{Minimisation of perceptual memory effects}

Binocular onset rivalry is known to be influenced by perceptual memory of immediately preceding images (Pearson \& Brascamp, 2008); this may manifest in the present investigation as a 'successive fear effect', where both trial ${ }_{i}$ and trial $l_{i+1}$ are perceived as "fear". We assessed this effect in Experiment 2 in order to minimise the potentially confounding effects of perceptual memory on our experimental manipulation and excluding participants who showed a strong influence of perceptual memory in their responding. A mean of $11.60 \%(s d=7.74 \%)$ of all trials showed a successive fear effect, where both trial ${ }_{i}$ and trial $l_{i+1}$ were reported as fear. One participant demonstrated this effect to a greater extent than all others (> group mean +3 sd at $42.95 \%$ ), and was excluded from analysis in Experiments 2 . We also assessed whether individual fear faces were exclusively classified as fear for 
each participant, suggesting a perceptual memory of fear for a specific KDFE model which persisted throughout the experiment. Across all participants and image pairs, fear faces showed a mean dominance frequency of $32.46 \%$ ( $s d=16.69 \%)$. No image pairs showed a substantially greater dominance frequency than the mean for the stimulus set (all $<$ mean $+3 s d)$. In assessing each participant separately, a mean of two image pairs ( $s d=1.66$ image pairs) showed exclusive fear dominance for each participant (fear was reported in every presentation of those image pairs). Trails of image pairs with exclusive fear dominance within a single participant were removed from further analysis in Experiment 2.

\section{Reaction times}

For each participant, individual trials were excluded if the reaction time exceeded the participant mean reaction time \pm 3 sd of that mean. Reaction time data was not available for one participant due to equipment failure.

\section{Statistical analysis}

All data were normally distributed according the Shapiro-Wilk test statistic $(p>.05)$. To assess differences in the onset bias of fearful over neutral faces when presented at threshold sensitivity levels (Experiment 2), the percentage of fear faces reported at each cardiac phase was entered into a two-way mixed ANCOVA with the between subjects factor "interoceptive accuracy" (low, high) and covariates of state anxiety, and the difference in fear sensitivity between systole and diastole (systole - diastole). Reaction time data for Experiment 2 were also entered into a $2 \times 2$ mixed ANCOVA, with the within subjects factors "cardiac cycle" (systole, diastole) and "emotion reported" (fear, neutral), the between subject factor "interoceptive accuracy" (low, high) and covariates state anxiety, and the difference in fear sensitivity between systole and diastole (systole - diastole). 


\section{Results and discussion}

There was a mean fear categorisation of $25.28 \%$, collapsed across participants and cardiac conditions (mean systole $=25.43 \%$, mean diastole $=25.12 \%$; all participants were within the range of mean \pm 3 sd). A one-down-one-up staircase using a ratio of $\delta$-/ $\delta+$ of 0.2845 has been shown in modelling simulations to converge on a detection rate of $22.15 \%$ when the size $\delta+$ of a step up is larger than half the spread of the psychometric function (García-Pérez, 1998). Given that the spread of the psychometric function of this manipulation has not previously been explored, a mean fear detection rate of $25.28 \%$ collapsed across both conditions was taken to suggest the staircase manipulation had been successful in converging towards the modelled detection rate and thereby reversing the onset bias of fearful over neutral faces reported elsewhere (e.g. Alpers \& Gerdes, 2007).

There was no main effect of cardiac phase on the frequency of fear categorisations $(F(1,23)=0.01, p$ $\left.=.974, \eta_{p}^{2}<.00\right)$ and no evidence for the predicted significant interaction between cardiac phase and interoceptive accuracy in the frequency of fear categorisation (high interoceptive accuracy group: mean systole fear detection $=26.25 \%(s d=10.80 \%)$, mean diastole fear detection $=24.75 \%(s d=$ 9.12\%); low interoceptive accuracy group: mean systole fear detection $=23.53 \%$ (sd $=7.04 \%$ ), mean diastole fear detection $=24.25 \%(s d=6.54 \%) ; F(1,23)=1.50, p=.233, \eta_{p}^{2}=.06$, Figure 3B $)$. There were no further significant main effects or interactions (all $p>.05$ ).

In exploratory analysis of reaction times, across the whole group cardiac phase did not differentially modulate the time required to respond to the emotion classification task $(F(1,23)=1.44, p=.242$, $\left.\eta_{p}^{2}=.06\right)$. However as in Experiment 1, there was a significant interaction between cardiac phase and interoceptive accuracy $\left(F(1,23)=7.368, p=.012, \eta_{p}^{2}=.24\right)$, evident as a significant difference in reaction times to perform emotional judgements (collapsed across fear and neutral) at systole and diastole between high and low interoceptive accuracy groups (high interoceptive accuracy group: mean systole reaction time $=1069.07 \mathrm{~ms}(\mathrm{sd}=439.01 \mathrm{~ms})$, mean diastole reaction time $=1107.61$ 
$\mathrm{ms}(\mathrm{sd}=469.93 \mathrm{~ms}$ ); low interoceptive accuracy group: mean systole reaction time $=1060.24 \mathrm{~ms}$ (sd $=351.08 \mathrm{~ms})$, mean diastole reaction time $=1038.86 \mathrm{~ms}(\mathrm{sd}=355.45 \mathrm{~ms})$, mean group systole diastole difference in reaction time $=59.91 \mathrm{~ms}, 95 \% \mathrm{Cl}[17.16,102.70] ;\left(t(25)=2.89, p=.008, \eta_{p}^{2}=\right.$ .25 , Figure $3 C)$. Individuals with high interoceptive accuracy had reduced reaction times in performing the emotional categorisation task at systole compared to diastole (mean systole diastole difference $=-41.22 \mathrm{~ms}, 95 \% \mathrm{Cl}[-73.72,-8.72])$. In contrast, individuals with low interoceptive accuracy show a non-significant increase in reaction times to perform the emotional categorisation task at systole compared to diastole (mean systole - diastole difference $=24.26 \mathrm{~ms}$, $95 \% \mathrm{Cl}[-9.63,58.15])$. There were no significant main effects of the emotion identified, nor interactions between emotion, state anxiety and interoception (all $p>.05$ ). These data suggest that cardiac phase and interoceptive accuracy do not bias the overall tendency to categorise emotionally ambiguous faces as fearful, however they do interact to modulate the speed at which these emotional judgements are performed for both fear and neutral decisions, such that individuals with high interoceptive accuracy were faster to perform an emotion categorisation judgements at systole relative to diastole. 
A

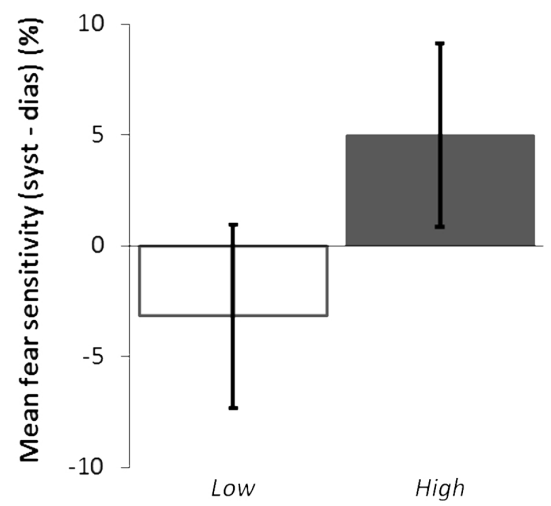

Interoceptive accuracy

B

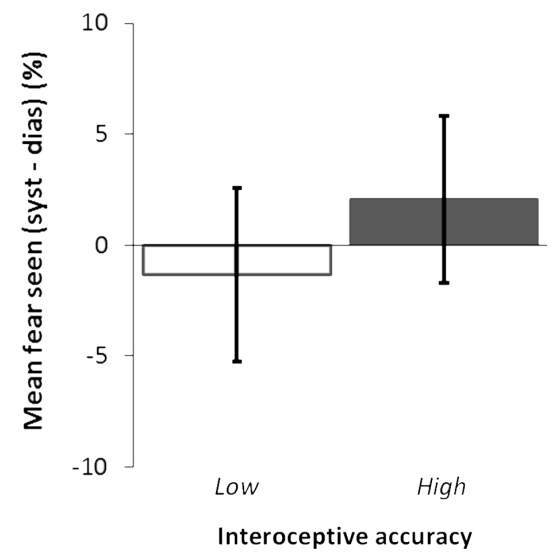

C

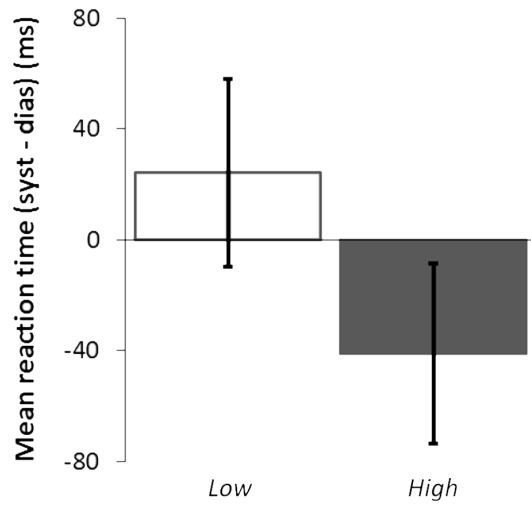

Interoceptive accuracy

Figure 3. Interactions between interoceptive accuracy and (A) fear sensitivity (Experiment 1), (B-C) emotion categorisation task performance (Experiment 2). (A) Individuals with high interoceptive accuracy show increased fear sensitivity at systole relative to diastole. (B) Systole and diastole presentations did not bias the frequency of fear responding for high or low interoception groups. (C) Individuals with high interoceptive accuracy were faster to perform the emotion categorisation task (collapsed across fear and neutral judgements) at systole relative to diastole. All error bars $=95 \% \mathrm{Cl}$. 


\section{General discussion}

Interoceptive processes influence percept formation for fear faces. Using a binocular onset rivalry paradigm, we show the effects of cardiac afferent signalling and interoceptive ability in shaping rapid percept formation and conscious access of emotionally-salient fearful and neutral face images. Individuals with high interoceptive accuracy were more sensitive to the detection of fearful faces when they were presented at systole relative to diastole, while individuals with low interoceptive accuracy were less sensitive at systole relative to diastole (Experiment 1, Figure 3A). These effects were mirrored in reaction time data: Individuals with high interoceptive accuracy were faster to perform an emotion categorisation judgement at systole relative to diastole, but individuals with low interoceptive accuracy were slower at systole relative to diastole (Experiment 2, Figure 3C). Importantly, there was no overall difference in the tendency to categorise ambiguous faces as fearful at systole or diastole for either group (Figure 3B). This indicates that, for this task, baroreceptor activation does not lead to an overall response bias towards fear responding, but rather supports the detection of subtle fear signals and promotes rapid decision making in categorising ambiguous emotions for individuals with a high 'constitutional' level of interoceptive accuracy. Our task appropriately approximates perceptual rivalry encountered in normal vision (Stanley et al., 2011), hence our findings elucidate how naturalistic visual competition involving affective stimuli is subject to interacting influences of bottom-up interoceptive (baroreceptor) signalling and an individual's characteristic interoceptive ability. Our data, from two separate experiments, therefore highlight the role of interoceptive and visceral bodily dimensions in shaping emotional responses.

Previous work suggests that the perceived intensity of fearful stimuli is increased by bottom-up interoceptive effects of baroreceptor activation at cardiac systole (Garfinkel et al., 2014). We therefore hypothesised that, under conditions of binocular onset rivalry, fearful faces would be detected at lower contrasts during systole compared to diastole, consistent with sensitisation to 
fearful stimuli during baroreceptor activation. In line with our hypothesis, sensitivity to fear faces was increased at systole relative to diastole, but interestingly only in participants with high interoceptive accuracy (Figure 3A). This finding suggests that the inferential processes involved in the conscious access of emotional faces are indeed influenced by the bottom-up visceral state (presence and absence of baroreceptor activation) and sensitivity to these states. Specifically, systole has a sensitizing effect to fear stimuli for those with greater conscious access to cardiac interoceptive signals.

To date, reported effects of baroreceptor activation include observed suppression of nociceptive processing (Edwards et al., 2002), suppression of memory encoding (Garfinkel et al., 2013), and the enhanced processing of fear and threat signals (Garfinkel et al., 2014) (to a lesser extent also disgust stimuli (M. A. Gray et al., 2012)). Our analysis of both behavioural sensitivity in the detection of low contrast fearful faces and reaction times during emotional categorisation suggest that cardiac afferent signals and interoceptive abilities modulate the conscious access of emotionally salient stimuli. This effect may be understood in terms of perceptual inference informed by the contextual cues of interoceptive responses (Ainley, Apps, Fotopoulou, \& Tsakiris, 2016; Allen et al., 2016; Seth \& Critchley, 2013). Individuals with high interceptive accuracy may place more weight (higher attention or 'expected precision') on interoceptive signals when forming perceptual inferences about emotional stimuli, leading to the significant cardiac phase effects observed for these individuals ("High" group in Figures 2 and 3B). Conversely there is less influence (lower expected precision) of interoceptive signals on perceptual inference for individuals with low interoceptive accuracy ("Low" group in Figures 2 and 3B). We further demonstrate that, in the overall biasing effects of cardiac timing (Experiment 2, Figure 3B), individuals with high interoceptive accuracy do not show an increased tendency to report the detection of fear faces, rather they show a specific facilitation in detecting fear stimuli (Experiment 1) and in making emotional judgements (Experiment 2) in low visual signal strength conditions when the stimulus co-occurs with interoceptive afferent activation. The current findings are in line with previous work demonstrating enhanced stimulus sensitivity for 
fear faces using a cardiac emotional attentional blink paradigm. Stimulus "breakthrough" of faces is enhanced when time-locked to cardiac systole relative to diastole, an effect that manifested specifically for fear faces (Garfinkel et al., 2014). In addition to these phase effects of cardiac cycle affecting stimulus sensitivity, previous work has shown that other features of cardio-stimulus coupling can affect visual experience. Stimuli repeatedly presented with the same frequency as the cardiac cycle take longer to access visual awareness and are also more difficult to discriminate (Salomon et al., 2016). In a series of experiments where stimuli were presented synchronous to the cardiac cycle (phase locked to systole or diastole) or presented asynchronously (faster / slower), cardio-coupling reduced stimulus processing. The robustness of this effect has been shown using both continuous flash suppression (CSF) and visual crowding paradigms (Salomon et al., 2016), thus highlighting a specific role of temporal based cardiac-stimulus coupling that can manifest independent of cardiac phase. In contrast to the present study, the reduced effect of stimulus processing driven by temporal alignment with the cardiac phase was not found to be influenced by individual differences in interoceptive accuracy (Salomon et al., 2016). Taken together, these different results point to distinct phase and temporal effects of cardio-stimulus synchronicity that impact stimulus processing.

Our findings give empirical support for predictions from peripheral models of emotion which propose the origin of emotional feelings to be the perception of changes within the body (Lange \& James, 1967). It is observed that individuals with high interoceptive accuracy can better guess whether a shock would be delivered following an unseen (masked) stimulus in an unconscious 'trace' fear conditioning paradigm (Katkin, Wiens, \& Ohman, 2001). The interpretation is that these individuals use their greater sensitivity to visceral signals to detect internal physiological changes, evoked preconsciously by threat, to inform emotional judgements. More recently it has been shown that interoceptive signals within the brain may also enhance detection of non-emotional stimuli, for example the magnitude of a heartbeat evoked potential predicts visual detection of faint visual gratings at the threshold of conscious experience (Park, Correia, Ducorps, \& Tallon-Baudry, 2014). 
These and similar observations (e.g. in the respiratory domain (Zelano et al., 2016) link sensory signals to the strength of afferent visceral representations.

In the present study, using a binocular onset rivalry paradigm, we take advantage of the phasic nature of baroreceptor activation to demonstrate that visceral afferent signals at the level of individual heartbeats influence perceptual inference during resolution of competing binocular stimuli. In a novel experimental paradigm, we extend the description of the sensitizing effects of cardiac systole for fear stimuli (Garfinkel et al., 2014) to demonstrate a circumstance in which the impact of baroreceptor activation on fear processing is stratified across individuals according to their performance on heartbeat detection tests of interoceptive accuracy. In this context, interoceptive accuracy is viewed as a largely invariant 'constitutional' individual difference that indicates a stronger representation of internal bodily state. Together our findings support recent models of body-brain interaction (Critchley \& Nagai, 2012; Park, Tallon-baudry, \& B, 2014; Seth, 2013) along with contextualized, predictive coding accounts of emotion processing (Seth \& Critchley, 2013; Seth, Suzuki, \& Critchley, 2012), which extend our understanding of the important contribution of interoception to fear and threat processing. 


\section{References}

Ainley, V., Apps, M. A. J., Fotopoulou, A., \& Tsakiris, M. (2016). 'Bodily precision': a predictive coding account of individual differences in interoceptive accuracy. Philosophical Transactions of the Royal Society of London B: Biological Sciences, 371(1708).

Allen, M., Frank, D., Schwarzkopf, D. S., Fardo, F., Winston, J. S., Hauser, T. U., ... Sigman, M. (2016). Unexpected arousal modulates the influence of sensory noise on confidence. ELife, 5, 403-450. https://doi.org/10.7554/eLife.18103

Alpers, G. W., \& Gerdes, A. B. M. (2007). Here is looking at you: emotional faces predominate in binocular rivalry. Emotion (Washington, D.C.), 7(3), 495-506. https://doi.org/10.1037/15283542.7.3.495

Alpers, G. W., \& Pauli, P. (2006). Emotional pictures predominate in binocular rivalry. Cognition \& Emotion, 20(5), 596-607. Retrieved from http://www.tandfonline.com/doi/abs/10.1080/02699930500282249\#.Vakwf_IVhBc

Blake, R., \& Logothetis, N. K. (2002). Visual Competition. Nature Reviews Neuroscience, 3(1), 13-21. https://doi.org/10.1038/nrn701

Carter, O., \& Cavanagh, P. (2007). Onset Rivalry: Brief Presentation Isolates an Early Independent Phase of Perceptual Competition. PLoS ONE, 2(4), e343. https://doi.org/10.1371/journal.pone.0000343

Chong, S. C., \& Blake, R. (2006). Exogenous attention and endogenous attention influence initial dominance in binocular rivalry. Vision Research, 46(11), 1794-1803. https://doi.org/10.1016/j.visres.2005.10.031

Critchley, H. D., Eccles, J., \& Garfinkel, S. N. (2013). Interaction between cognition, emotion, and the 
autonomic nervous system. Handbook of Clinical Neurology (1st ed., Vol. 117). Elsevier B.V. https://doi.org/10.1016/B978-0-444-53491-0.00006-7

Critchley, H. D., \& Nagai, Y. (2012). How Emotions Are Shaped by Bodily States. Emotion Review, 4(2), 163-168. https://doi.org/10.1177/1754073911430132

Dunn, B. D., Stefanovitch, I., Evans, D., Oliver, C., Hawkins, A., \& Dalgleish, T. (2010). Can you feel the beat? Interoceptive awareness is an interactive function of anxiety- and depression-specific symptom dimensions. Behaviour Research and Therapy, 48(11), 1133-1138. https://doi.org/10.1016/j.brat.2010.07.006

Edwards, L., McIntyre, D., Carroll, D., Ring, C., \& Martin, U. (2002). The human nociceptive flexion reflex threshold is higher during systole than diastole. Psychophysiology, 39(5), 678-681. https://doi.org/10.1017.S0048577202011770

Edwards, L., Ring, C., Mclntyre, D., Winer, J. B., \& Martin, U. (2009). Sensory detection thresholds are modulated across the cardiac cycle: evidence that cutaneous sensibility is greatest for systolic stimulation. Psychophysiology, 46(2), 252-256. https://doi.org/10.1111/j.14698986.2008.00769.x

García-Pérez, M. A. (1998). Forced-choice staircases with fixed step sizes: asymptotic and smallsample properties. Vision Research, 38(12), 1861-1881. Retrieved from http://www.ncbi.nlm.nih.gov/pubmed/9797963

Garfinkel, S. N., Barrett, A. B., Minati, L., Dolan, R. J., Seth, A. K., \& Critchley, H. D. (2013). What the heart forgets: Cardiac timing influences memory for words and is modulated by metacognition and interoceptive sensitivity. Psychophysiology, 50(6), 505-512. Retrieved from http://www.pubmedcentral.nih.gov/articlerender.fcgi?artid=4340570\&tool=pmcentrez\&rende rtype=abstract 
Garfinkel, S. N., \& Critchley, H. D. (2016). Threat and the Body: How the Heart Supports Fear Processing. Trends in Cognitive Sciences, 20(1), 34-46. https://doi.org/10.1016/j.tics.2015.10.005

Garfinkel, S. N., Minati, L., Gray, M. A., Seth, A. K., Dolan, R. J., \& Critchley, H. D. (2014). Fear from the Heart: Sensitivity to Fear Stimuli Depends on Individual Heartbeats. Journal of Neuroscience, 34(19), 6573-6582. https://doi.org/10.1523/JNEUROSCI.3507-13.2014

Garfinkel, S. N., Seth, A. K., Barrett, A. B., Suzuki, K., \& Critchley, H. D. (2015). Knowing your own heart: Distinguishing interoceptive accuracy from interoceptive awareness. Biological Psychology, 104, 65-74. https://doi.org/10.1016/j.biopsycho.2014.11.004

Gendron, M., \& Barrett, L. F. (2009). Reconstructing the Past: A Century of Ideas About Emotion in Psychology. Emotion Review : Journal of the International Society for Research on Emotion, 1(4), 316-339. https://doi.org/10.1177/1754073909338877

Giles, N., Lau, H., \& Odegaard, B. (2016). What Type of Awareness Does Binocular Rivalry Assess? Trends in Cognitive Sciences, 20(10), 719-720. https://doi.org/10.1016/j.tics.2016.08.010

Gray, K. L. H. H., Adams, W. J., \& Garner, M. (2009). The influence of anxiety on the initial selection of emotional faces presented in binocular rivalry. Cognition, 113(1), 105-110. https://doi.org/10.1016/j.cognition.2009.06.009

Gray, M. A., Beacher, F. D., Minati, L., Nagai, Y., Kemp, A. H., Harrison, N. A., \& Critchley, H. D. (2012). Emotional appraisal is influenced by cardiac afferent information. Emotion (Washington, D.C.), 12(FEBRUARY), 180-191. https://doi.org/10.1037/a0025083

Hart, N., McGowan, J., Minati, L., \& Critchley, H. D. (2013). Emotional regulation and bodily sensation: interoceptive awareness is intact in borderline personality disorder. Journal of Personality Disorders, 27(4), 506-518. https://doi.org/10.1521/pedi_2012_26_049 
Katkin, E. S., Wiens, S., \& Ohman, a. (2001). Nonconscious fear conditioning, visceral perception, and the development of gut feelings. Psychological Science : A Journal of the American Psychological Society / APS, 12(5), 366-370. https://doi.org/10.1111/1467-9280.00368

Lange, C., \& James, W. (1967). The Emotions (1922). New York, USA: Hafner.

Leopold, D. A., Wilke, M., Maier, A., \& Logothetis, N. K. (2002). Stable perception of visually ambiguous patterns. Nature Neuroscience, 5(6), 605-609. https://doi.org/10.1038/nn0602-851

Lundqvist, D., Flykt, A., \& Ohman, A. (1998). The Karolinska Directed Emotional Faces - KDEF, CD ROM from Department of Clinical Neuroscience, Psychology section. Karolinska Institutet, ISBN 91-630-7164-9.

Miles, W. R. (1930). Ocular Dominance in Human Adults. The Journal of General Psychology, 3(3), 412-430. https://doi.org/10.1080/00221309.1930.9918218

Park, H.-D., Correia, S., Ducorps, A., \& Tallon-Baudry, C. (2014). Spontaneous fluctuations in neural responses to heartbeats predict visual detection. Nature Neuroscience, 17(4), 612-618. Retrieved from http://dx.doi.org/10.1038/nn.3671

Park, H.-D., Tallon-baudry, C., \& B, P. T. R. S. (2014). The neural subjective frame: from bodily signals to perceptual consciousness. Philosophical Transactions of the Royal Society of London. Series B, Biological Sciences, 369(1641), 20130208. https://doi.org/10.1098/rstb.2013.0208

Pearson, J., \& Brascamp, J. (2008). Sensory memory for ambiguous vision. Trends in Cognitive Sciences, 12(9), 334-341. https://doi.org/10.1016/j.tics.2008.05.006

Pfeifer, G., Garfinkel, S. N., Gould van Praag, C. D., Sahota, K., Betka, S., \& Critchley, H. D. (2017). Feedback from the heart: Emotional learning and memory is controlled by cardiac cycle, interoceptive accuracy and personality. Biological Psychology, 126, 19-29. https://doi.org/10.1016/J.BIOPSYCHO.2017.04.001 
Salomon, R., Ronchi, R., Donz, J., Bello-Ruiz, J., Herbelin, B., Martet, R., ... Blanke, O. (2016). The Insula Mediates Access to Awareness of Visual Stimuli Presented Synchronously to the Heartbeat. Journal of Neuroscience, 36(18), 5115-5127. https://doi.org/10.1523/JNEUROSCI.4262-15.2016

Schandry, R. (1981). Heart beat perception and emotional experience. Psychophysiology, 18(4), 483488. https://doi.org/10.1111/j.1469-8986.1981.tb02486.x

Seth, A. K. (2013). Interoceptive inference, emotion, and the embodied self. Trends in Cognitive Sciences, 17(11), 565-573. https://doi.org/10.1016/j.tics.2013.09.007

Seth, A. K., \& Critchley, H. D. (2013). Extending predictive processing to the body: Emotion as interoceptive inference. Behavioral and Brain Sciences, 36(03), 227-228. https://doi.org/10.1017/S0140525X12002270

Seth, A. K., Suzuki, K., \& Critchley, H. D. (2012). An Interoceptive Predictive Coding Model of Conscious Presence. Frontiers in Psychology, 2, 395. https://doi.org/10.3389/fpsyg.2011.00395

Sherrington, C. S. (1948). The integrative action of the nervous system. Cambridge, UK: Cambridge University Press.

Song, C., Yao, H., Qian, N., Wheatstone, C., Blake, R., Logothetis, N., ... Spillmann, L. (2009). Duality in Binocular Rivalry: Distinct Sensitivity of Percept Sequence and Percept Duration to Imbalance between Monocular Stimuli. PLOS ONE, 4(9), e6912. https://doi.org/10.1371/journal.pone.0006912

Spielberger, C. D., Gorsuch, R. L., Lushene, R., Vagg, P. R., \& Jacobs, G. A. (1983). Manual for the State-Trait Anxiety Inventory (2nd ed.). Palo Alto, CA: Consulting Psychologists Press.

Stanley, J., Forte, J. D., Cavanagh, P., \& Carter, O. (2011). Onset rivalry: the initial dominance phase is independent of ongoing perceptual alternations. Frontiers in Human Neuroscience, 5(10), 140. 
https://doi.org/10.3389/fnhum.2011.00140

Tong, F., Meng, M., \& Blake, R. (2006). Neural bases of binocular rivalry. Trends in Cognitive Sciences, 10(11), 502-511. https://doi.org/10.1016/j.tics.2006.09.003

Wiens, S., Mezzacappa, E. S., \& Katkin, E. S. (2000). Heartbeat detection and the experience of emotions. Cognition \& Emotion, 14(3), 417-427. https://doi.org/10.1080/026999300378905

Zelano, C., Jiang, H., Zhou, G., Arora, N., Schuele, S., Rosenow, J., \& Gottfried, J. A. (2016). Nasal Respiration Entrains Human Limbic Oscillations and Modulates Cognitive Function. Journal of Neuroscience, 36(49). 


\section{Author Contributions}

C.D. Gould van Praag, G. Hassan, S.N. Garfinkel and R.B. Scott contributed to the study design. R. Scott, C. D. Gould van Praag and G. Hassan developed the experimental stimulus. G. Hassan carried out the experiments under the supervision of S. N. Garfinkel. C. D. Gould van Praag and S. N.

Garfinkel conducted data analysis. C. D. Gould van Praag, G. Hassan and S. N. Garfinkel drafted the manuscript. All authors made final comments on the manuscript.

\section{Acknowledgements}

We thank European Research Council, the Dr. Mortimer and Theresa Sackler Foundation and the University of Sussex for financial contributions. 